\title{
ANTIOXIDANT EFFECT OF RESVERATROL IN HUMAN SPERMATOZOA AND
}

\section{IN RAT GERMINAL CELLS}

\author{
Federico $\mathrm{MG}^{1}$, Collodel $\mathrm{G}^{1,2}$, Renieri $\mathrm{T}^{1,2}$, Pascarelli NA ${ }^{1}$, Geminiani $\mathrm{M}^{1}$, Martini $\mathrm{S}^{3}$, \\ Bonechi $C^{3}$, Rossi $C^{3}$, Moretti $E^{1,2}$ \\ ${ }^{1}$ Department of Biomedical Sciences, Applied Biology Section; \\ ${ }^{2}$ Interdepartmental Centre for Research and Therapy of Male Infertility; \\ ${ }^{3}$ Department Pharmaco-Chemical-Technological, University of Siena.
}

\begin{abstract}
Objective: To assess the antioxidant activity of Resveratrol (3,5,4'-trihydroxystilbene, RES) after induction of lipid peroxidation (LPO) in human spermatozoa and in immature rat germinal cells. Materials and Methods: Ejaculated human spermatozoa, selected by swim up, have been incubated with tert-Butylhydroperoxide and tert-Butylhydroperoxide-RES. The localization of LPO has been performed using the probe C11-BODIPY ${ }^{581 / 591}$. The same assays were carried out on pachytene spermatocytes and round spermatids obtained from three Wistar rats 35 days of age. The two cellular fractions were achieved after enzymatic digestion with collagenase and subsequent fractionation on bovine serum albumin 0.5-3\% gradient (STAPUT). The ultrastructure of all samples was assessed by transmission electron microscopy (TEM). Results: The midpiece of sperm tail and the whole plasma membrane of germ cells were the target of LPO. TEM analysis of sperm, quantitatively elaborated by a mathematical formula, showed a significantly lower percentage of necrosis in the samples treated with RES (P<0.01); as regards rat germinal cells, necrosis features (cytoplasmic vacuoles, disrupted chromatin and broken plasma membrane) were mainly evident in the meiotic fraction without RES. Conclusions: RES, found in the skins of grape, reduces the damage induced by oxidative stress in human sperm and rat testicular germ cells; in particular spermatids appeared to be less sensitive to oxidative damage compared with spermatocytes.
\end{abstract}

Key words: Germinal cells, Oxidative Stress, Resveratrol, Spermatozoa, STAPUT, TEM.

\section{INTRODUCTION}

Reactive oxygen species (ROS) play a functional role as second messengers in many cell types. When they are produced at a very low concentration, they trigger cell signaling events and regulate physiological function (1). Mammalian spermatozoa usually produce ROS, which support some main sperm functions such as capacitation, acrosome reaction, zona pellucida binding, and oocyte fusion (2). Nevertheless, uncontrolled ROS production can also play an important role in causing sperm aberration, leading to infertility. What is usually called "oxidative stress" appears to be the resultant of defects in the balance between the concentration of ROS and antioxidant scavenging system. Spermatozoa membranes are rich in polyunsaturated fatty acid and this makes them very susceptible to oxygen-induced damage, mediated by lipid peroxidation (LPO) (3). Oxidative stress has been suggested to be an important factor in the etiology of poor sperm function through peroxidative damage to the cell membrane, to DNA inducing single- and double-strand DNA breaks and to proteins (4-6). Spermatozoa and seminal plasma contain enzymes such as the glutathione peroxidase/reductase system, superoxide dismutase, catalase and low-molecular weight antioxidants, vitamin $\mathrm{E}$, vitamin $\mathrm{C}$, urate, albumin, that scavenge ROS in order to prevent possible cellular damage (7). One of the rational strategies to counteract the oxidative stress is to increase the scavenging capacity of seminal plasma. However, the use of antioxidant supplementation to reverse the effect of ROS is still debated $(8,9)$. In previous studies, the resveratrol $\left(3,5,4^{\prime}\right.$ trihydroxystilbene, RES), a natural phytoalexin, has exhibited a wide range of biological activities such as antiinflammatory, antioxidant, antiviral and antitumoral properties (10). Some recent in vivo studies in animal models demonstrated that RES play a positive effect even on male reproductive function (11-13). This study was designed to evaluate, in vitro, the protective effect of RES in ejaculated human spermatozoa and in rat germinal cells treated with tert-Butylhydroperoxide, an inductor of LPO. The oxidative damage in samples incubated with and without RES has been evaluated using the probe C11-BODIPY ${ }^{581 / 591}$ and transmission electron microscopy (TEM).

\section{MATERIAL AND METHODS}

Semen analysis and LPO induction and evaluation

Ten semen samples from men of proven fertility were collected by masturbation after 3-5 days of sexual abstinence and examined after liquefaction for $30 \mathrm{~min}$ at $37^{\circ} \mathrm{C}$. Volume, $\mathrm{pH}$, concentration and motility were evaluated according to World Health Organization guidelines (14). Motile sperm fraction was recovered 
by swim up technique as reported by Piomboni et al. (15) and then was incubated with $5 \mu \mathrm{M}$ of a fluorescent fatty acid probe, 4,4-difluoro-5-(4-phenyl-1,3-butadienyl)-4-bora-3a,4adiaza-s-indacene-3-undecanoic acid (C11-BODIPY ${ }^{581 / 591}$, Molecular Probes, Eugene, USA) at $37^{\circ} \mathrm{C}$ for $30 \mathrm{~min}$. Excess probe was removed by washing the cells twice with Biggers, Whitten, Whittingham medium for $10 \mathrm{~min}$, three times. At this point the sperm sample was divided in two aliquots, which were treated with tert-Butylhydroperoxide $(45 \mu \mathrm{M})$ and RES $(15 \mu M)$ or tert-Butylhydroperoxide without RES for 1 hour at $37^{\circ} \mathrm{C}$. C11-BODIPY ${ }^{581 / 591}$ is able to intercalate in the phospholipids bilayer. LPO was localized observing the changes in C11-BODIPY ${ }^{581 / 591}$ fluorescence. The intact probe fluoresces red when it is intercalated into the membrane $(\lambda$ excitation $=590$ and $\lambda$ emission $=635 \mathrm{~nm})$, and it shifts to green $(\lambda$ excitation $=485$ and $\lambda$ emission $=535 \mathrm{~nm}$ ) after oxidative radicals attack. The staining was examined by Leitz Aristoplan Microscope fluorescence (Leica, Wetzlar, Germany). The images were taken using Leica Q Fluoro Standard, Leica Chantal software. For each sperm sample, 200 cells were examined and the frequency of green cells was calculated. The examiner scored cells showing evident green stain.

\section{Transmission electron microscopy (TEM)}

Sperm morphology was evaluated by TEM. Samples were treated as reported by Baccetti et al. (16); TEM data were collected and mathematically elaborated $(16,17)$. This method is able to furnish the percentages of three main phenotypic sperm pathologies: immaturity, necrosis and apoptosis.

\section{Rat germ cells isolation}

Pachytene spermatocytes and round spermatids were obtained by three 35-day old Wistar rats (100 gr of body weight) (Charles River, Calco, Italy). Rats were sacrificed by cervical dislocation and testes were collected and placed in Minimum Essential Medium supplemented with pyruvate $(1 \mathrm{mM})$ and lactate. After removal of the tunica albuginea, the testes were placed in culture medium containing collagenase (activity $0.450 \mathrm{unity} / \mathrm{ml}$ ) and incubate for $10 \mathrm{~min}$ to remove interstitium that was discarded. A second incubation in a shaking water bath at $32^{\circ} \mathrm{C}$ for 45 min with the same enzymes and $0.1 \%$ Bovine Serum Albumin and DNAse was performed to partially digest the basal lamina of the tubules. The cell suspension obtained after enzymatic digestion was centrifuged and fractionated by velocity sedimentation at unit gravity on $0.5-3 \%$ albumin gradient (STAPUT method) (18). The collected purified cellular fractions are made up by pachytene spermatocytes and spermatids; these fractions were identified on the basis of their morphological features by light microscopy and TEM. Cellular fractions were processed as described for human sperm.

\section{Statistical ANALYsis}

Statistical analysis was performed using SPSS v.13.0 Software (SPSS Inc., Chicago, IL). The KolmogorovSmirnov test was used to verify a normal or non-normal distribution of values. Because non-normal distribution of the values, the Mann Whitney test was used to compare the analysed variables. $\mathrm{P}<0.05$ was considered to be significant.

\section{RESULTS}

After treatment by tert-Butylhydroperoxide, the localization of LPO, detected by the C11-BODIPY $581 / 591$ probe, was not equally distributed over the sperm membrane but occurs mainly in the midpiece region of human spermatozoa (Fig. 1 B). The percentage of LPO (Table 1$)$ is significantly lower $(\mathrm{P}<0.01)$ in sperm samples treated with RES (Fig. ID) compared to that observed in samples without RES (Fig 1B). TEM analysis

Table 1. Means \pm SD of sperm pathologies quantified by the formula of Baccetti et al. (16) and LPO detected by C11-BODIPY 35194 , in semen samples treated with tert-Butylhydroperoxide with and without RES.

\begin{tabular}{|c|c|c|c|c|c|c|c|c|}
\hline \multirow{2}{*}{ Cases } & \multicolumn{2}{|c|}{ Apoptosis \% } & \multicolumn{2}{|c|}{ Necrosis \% } & \multicolumn{2}{|c|}{ Immaturity \% } & \multicolumn{2}{|c|}{ LPO \% } \\
\hline & -RES & +RES & -RES & +RES & -RES & +RES & -RES & +RES \\
\hline Mean \pm SD & $6.18 \pm 0.85$ & $5.33 \pm 0.45$ & $50.69 \pm 5.21^{*}$ & $35.39: 8.43$ & $54.19 \div 1.60$ & $53.18 \pm 1.15$ & $94.3 \pm 2.71^{*}$ & $22.3 \pm 3.02$ \\
\hline${ }^{\circ}$ Mean \pm SD & \multicolumn{2}{|c|}{$4.06 \div 2.05$} & \multicolumn{2}{|c|}{$32.13 \pm 10.58$} & \multicolumn{2}{|c|}{$48.83 \pm 13.93$} & & \\
\hline
\end{tabular}

$* \mathrm{P}<0.01$

${ }^{\circ}$ Means \pm SD: percentage of sperm pathologies in sperm from men with proven fertility used as controls (17). 

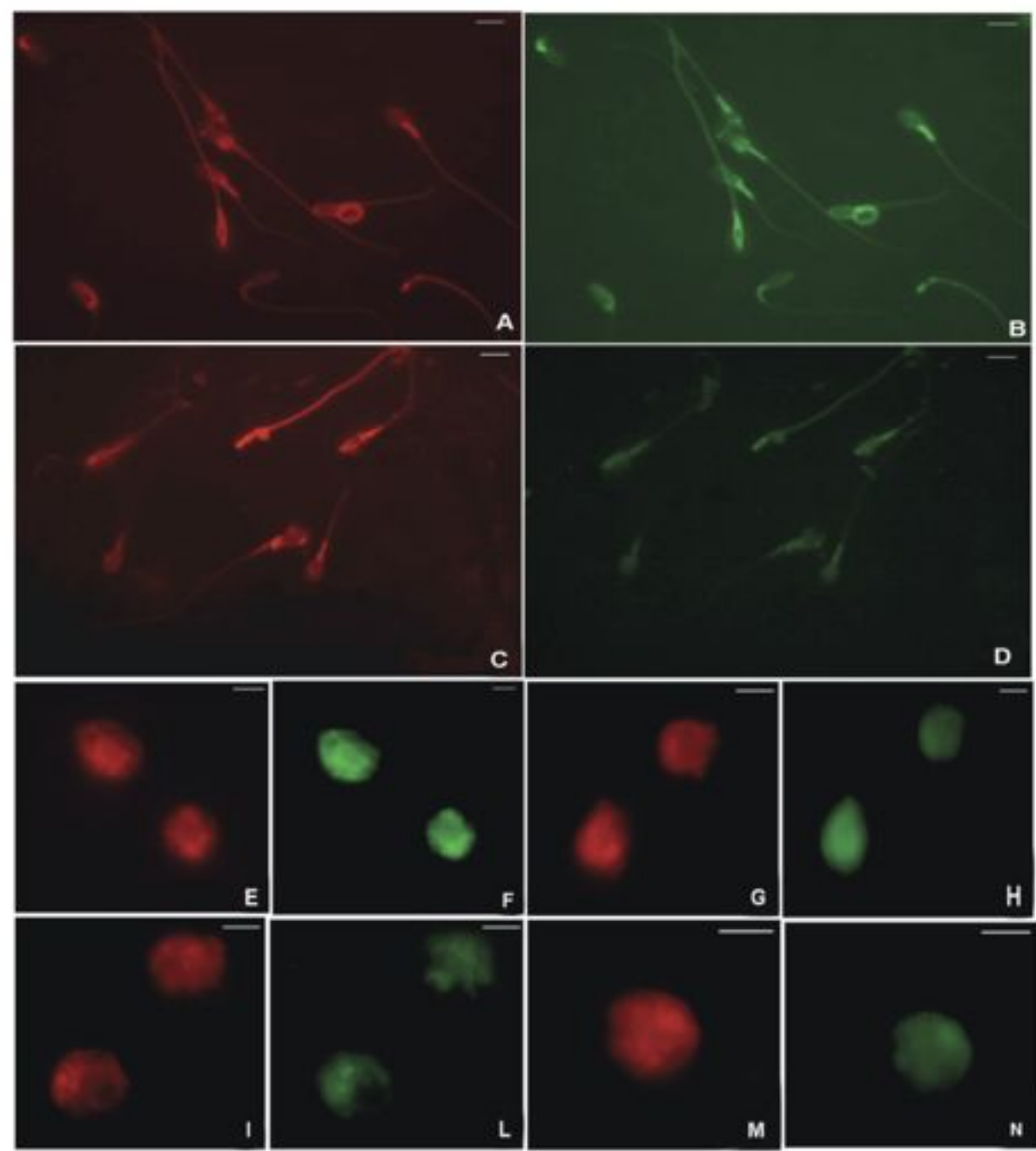

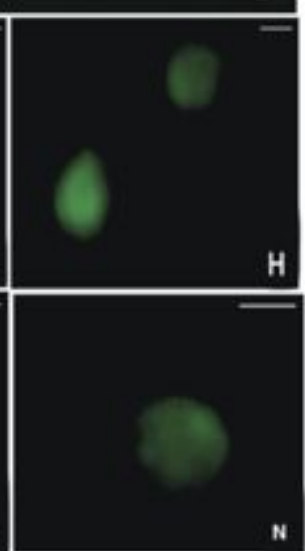

Fig.1:

Ultraviolet micrographs

of human sperm and rat germinal cells treated with tert-Butylhydroperoxide to induce LPO. The samples were incubated with and whitout RES and C11BODIPY ${ }^{581 / 591}$ probe was used to highlight LPO. For each sample two micrographs were acquired: the red one showing the intercalated probe in the membrane, the green one showing the intensity of peroxidation.

(A, B) Spermatozoa without RES.

(C, D) Spermatozoa with RES.

(E, F) Pachytene spermatocytes without RES. $(G, H)$ Pachytene spermatocytes with RES. (I, L) Spermatids without RES. $(M, N)$ Spermatids with RES.

Bar: $2 \mu m$. mathematically elaborated showed that the levels of necrosis were significantly higher $(\mathrm{P}<0.01)$ in sperm samples treated with tert-Butylhydroperoxide without RES than those incubated with RES (Table 1). The typical ultrastructural features of sperm necrosis are: broken plasma membrane, reacted or absent acrosome, misshaped nuclei with disrupted chromatin, poor axonemal and periaxonemal cytoskeletal structures. Isolated pachytene spermatocytes and round spermatids were treated with the same procedures used for sperm. In both cellular fractions we observed that the LPO localization was extended to the whole plasma membrane. In absence of RES, the damage induced by oxidative stress, as demonstrated by C11-BODIPY ${ }^{581 / 591}$ and TEM, was more evident in spermatocytes (Figs 1F, 2A) than spermatids (Figs 1L, 2C). The presence of RES attenuates the induction of LPO mainly in the meiotic fraction (Figs. 1H, 2B). The spermatids appeared to be more resistant to ROS attack: the damages induced by LPO are more evident by TEM (Figs 2C, D) than by fluorescence (Figs 1L, N).

\section{Discussion}

A variety of foods and beverages of vegetable origin contains several non-flavonoid classes of phenolic compounds synthesized by plants in response to injury or fungal attack. Among them, resveratrol has been iden- tified as the major active compound of stilbene phytoalexins and it is presumed to be beneficial for human health (10), mainly for its scavenger abilities against ROS. Trans-Resveratrol, the most common isomer, exhibits a protective effect against lipid peroxidation in cell membranes and DNA damage caused by ROS (11). In this study we demonstrated the protective effect of RES against LPO induced by $45 \mu \mathrm{M}$ tert-Butylhydroperoxide by means of C11-BODIPY ${ }^{581 / 591}$ probe, able to localize LPO, and TEM, a powerful tool for individuation of ultrastructural damages. For this purpose we used two cellular models: human spermatozoa, highly differentiated cells and immature germ cells from rat testis, separated in two fractions: meiotic fraction (pachytene spermatocytes) and postmeiotic fraction (spermatids). Our data showed that spermatid fraction is more resistant to LPO respect to pachytene spermatocyte. Mature spermatozoa, as previously showed (19), appeared to be subject to LPO only in the mitochondrial region, responsible of oxidative metabolism: the cytochromes in the respiratory chain, located in the inner mitochondrial membranes, contain iron that can accelerate sperm LPO. The head plasma membrane remained unperoxidized; the presence of a potent antioxidant system in the head of the sperm cell would appear to be an obvious explanation of this observation. In this respect, it is important to re- 
Fig.2:

TEM micrographs of cross sections of germinal cells treated with tert-Butylhydroperoxide with and without RES.

(A) Pachytene spermatocytes without RES showing nuclei $(N)$ with disrupted

chromatin; the plasma membranes are broken (arrows) and the cytoplasms are vacuolated.

(B) Pachytene spermatocyte treated with RES presents normal ultrastructure.

(C) Spermatids without RES: the left cells show normal ultrastructural characteristics, whereas the right cell appears necrotic with broken plasma membrane.

(D) Spermatid with RES; the cell is normal, the acrosome $(A)$ is evident. Bar $=1 \mu \mathrm{m}$
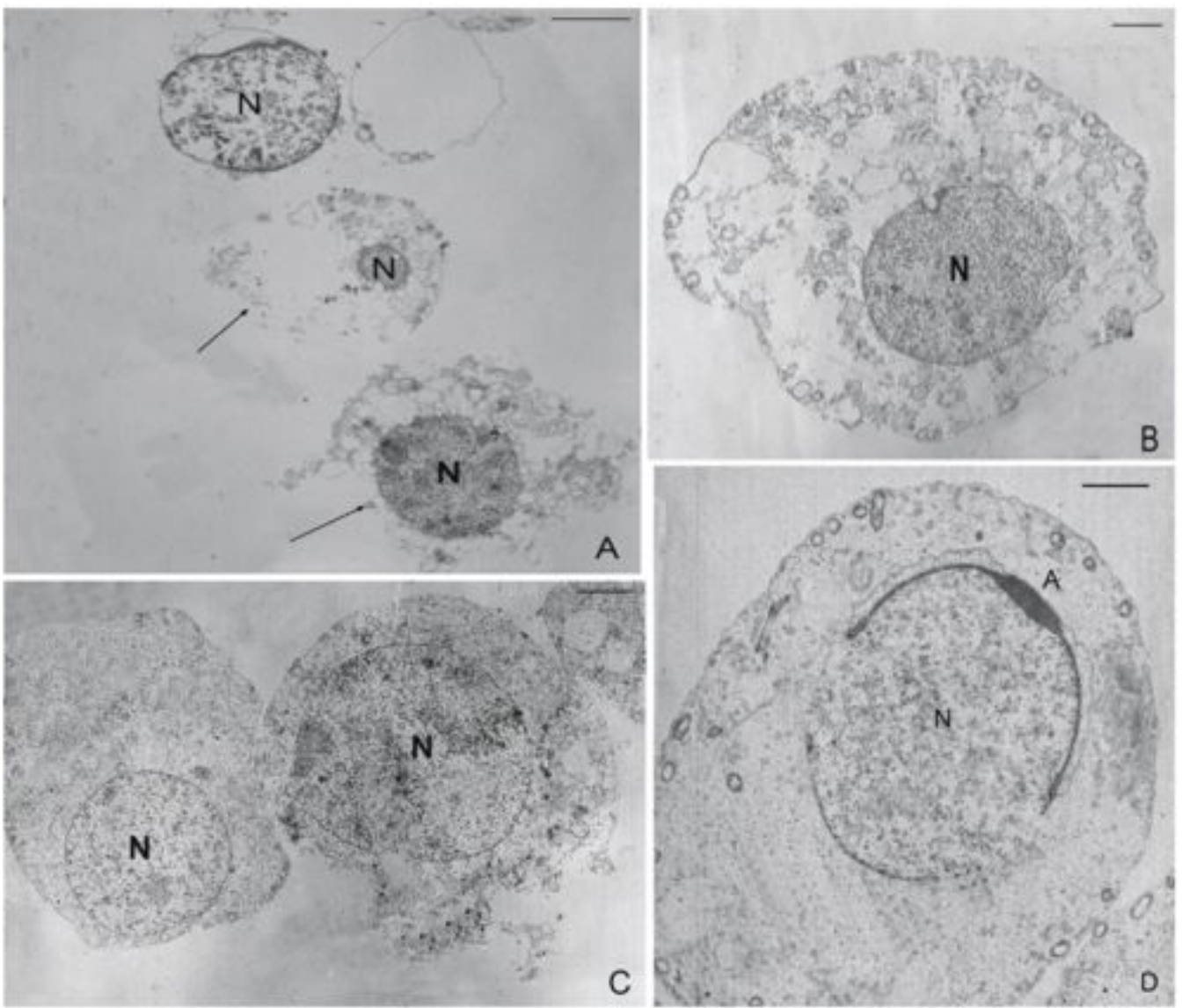

alize that the protection of the content of the sperm head, the paternal DNA, is of eminent importance, while the sperm mitochondria and tail will be eliminated once fertilization has occurred and may, therefore, be less protected against damage (20).

In conclusion, our results, although preliminary, are giving support to the evidence of antioxidant properties of RES against induced sperm LPO. Nevertheless, these studies cannot be extrapolated to the in vivo outcome, because the effect of RES supplementation on male infertility has not been yet explored.

\section{REFERENCES}

1- Forman HJ, Torres M. Reactive oxygen species and cell signaling: respiratory burst in macrophage signaling. Am J Respir Crit Care Med 2002;166: 4-8

2-de Lamirande E, Jiang H, Zini A, Kodama H, Gagnon C. Reactive oxygen species and sperm physiology. Rev Reprod 1997;2: 4854

3-Sikka SC. Relative impact of oxidative stress on male reproductive function. Curr Med Chem 2001;8: 851-862

4-Moustafa MH, Sharma RK, Thornton J, Mascha E, Abdel-Hafez MA, Thomas AJ Jr, Agarwal A. Relationship between ROS production, apoptosis and DNA denaturation in spermatozoa from patients examined for infertility. Hum Reprod 2004;19: 129-138 5-Stadtman ER, Levine RL. Free radical-mediated oxidation of free amino acids and amino acid residues in proteins. Amino Acids 2003;25: 207-218

6-Aitken RJ, De Luliis GN. Value of DNA integrity assays for fertility evaluation. Soc Reprod Fertil Suppl 2007;65: 81-92

7-Smith R, Vantman D, Ponce J, Escobar J, Lissi E. Total antioxidant capacity of human seminal plasma. Hum Reprod 1996;11: 1655-1660

8-Tremellen $K$. Oxidative stress and male infertility a clinical perspective. Hum Reprod Update 2008;14: 243-258
9-Agarwal A, Nallella KP, Allamaneni SS, Said TM. Role of antioxidants in treatment of male infertility: an overview of the literature. Reprod Biomed Online 2004;8: 616-627

10-Frèmont L. Biological effects of resveratrol. Life Sci 2000;66: 663-673

11-Juan ME, González-Pons E, Munuera T, Ballester J, RodríguezGil JE, Planas JM. trans-Resveratrol, a natural antioxidant from grapes, increases sperm output in healthy rats. J Nutr 2005;135: 757-760

12-Uguralp S, Usta U, Mizrak B. Resveratrol may reduce apoptosis of rat testicular germ cells after experimental testicular torsion. Eur J Pediatr Surg 2005;15: 333-336

13-Shin S, Jeon JH, Park D, Jang MJ, Choi JH, Choi BH, Joo SS, Nahm SS, Kim JC, Kim YB. trans-Resveratrol relaxes the corpus cavernosum ex vivo and enhances testosterone levels and sperm quality in vivo. Arch Pharm Res 2008;31: 83-87

14-WHO Laboratory Manual for the Examination of Human Semen and Semen-Cervical Mucus Interaction. Cambridge University, Cambridge 1999

15-Piomboni P, Bruni E, Capitani S, Gambera L, Moretti E, La Marca A, De Leo V, Baccetti B. Ultrastructural and DNA fragmentation analyses in swim-up selected human sperm. Arch Androl 2006;52: $51-59$

16-Baccetti B, Bernieri G, Burrini AG, Collodel G, Crisà N, Mirolli M, Moretti E, Piomboni P. Notulae seminologicae. 5. Mathematical evaluation of interdependent submicroscopic sperm alterations. $J$ Androl 1995;16: 365-371

17-Collodel G, Moretti E. Morphology and meiotic segregation in spermatozoa from men of proven fertility. J Androl 2008;29: 106114

18-Chiarella P, Puglisi R, Sorrentino V, Boitani C, Stefanini M. Ryanodine receptors are expressed and functionally active in mouse spermatogenic cells and their inhibition interferes with spermatogonial differentiation. J Cell Sci 2004;117(Pt18): 4127-4134

19-Cosci I, Moretti E, Colldel G. Lipid peroxidation in human spermatozoa from men with genitourinary infections. Syst Biol Reprod Med 2008;54: 75-83

20-Brouwers JF, Gadella BM. In situ localization of lipid peroxidation in individual bovine sperm cells. Free Radic Biol Med 2003;35: 1382-1391 